\title{
Baseline predictors of tolerability to carvedilol in patients with chronic heart failure
}

\author{
H Krum, D Ninio, P MacDonald
}

\begin{abstract}
Objective-To determine baseline predictors of tolerance to the $\alpha / \beta$ blocker carvedilol in everyday clinical practice.

Design-Retrospective analysis of tolerance to carvedilol in patients with chronic heart failure. Tolerance was defined as currently on carvedilol or on it at the time of death or heart transplantation. To meet the criteria for tolerance, carvedilol had to be prescribed at a stable dose for $\geqslant 3$ months.

Setting-Everyday clinical practice, comprising both hospital specialist practice and private practice. Tolerance was assessed in all patients prescribed carvedilol for chronic heart failure in those practices.

Patients-808 consecutive patients in both hospital specialist (611 patients) and private practice (197 patients).

Main outcome measures-Baseline predictors of tolerance assessed by proportional hazards analysis. Both univariate and multivariate analyses were performed.

Results-Within the entire cohort of 808 patients, 95 had stopped carvedilol, 606 were currently receiving the drug, 50 had died, and 44 had received a heart transplant. Overall, $88 \%$ of patients tolerated carvedilol ( $87 \%$ in the hospital specialist group, $92 \%$ in the private practitioner group). Factors that indicated impaired tolerance by univariate analysis were increased age in years (hazard ratio $1.01,95 \%$ confidence interval (CI) 1.0 to 1.3), low diastolic blood pressure (hazard ratio $1.04,95 \%$ CI 1.02 to 1.08 ), and raised plasma urea concentration (hazard ratio $1.04,95 \%$ CI 1.02 to 1.05 ). New York Heart Association (NYHA) class was also a marker of tolerance (proportion not tolerated: 3\% class I; 9\% class II; 13\% class III, 22\% class IV). By multivariate analysis, no single baseline variable was an independent marker of inability to tolerate carvedilol. Tolerance was also assessed in relation to traditional precautions or relative contraindications to $\beta$ blockade. Tolerance in these subgroups was: chronic obstructive airways disease/asthma $85 \%$ (89 patients), diabetes $86 \%$ (127 patients), peripheral vascular disease $84 \%$ (58 patients), concomitant amiodarone treatment $83 \%$ (230 patients), and heart rate $<70$ beats $/ \mathrm{min} 84 \%$ (184 patients).

Conclusions- $\beta$ Blocker treatment was well tolerated in everyday clinical practice, including non-hospital-based private practice. There was no single predictor of poor tolerance on multivariate analysis, although there was a clear association with NYHA class as well as age, diastolic blood pressure, and plasma urea on univariate analysis. Carvedilol was tolerated well among selected patients with traditional contraindications to $\beta$ blockade in this situation.
\end{abstract}

(Heart 2000;84:615-619)

Keywords: chronic heart failure; $\beta$ blockade; carvedilol

Clinical Pharmacology

Unit, Department of

Epidemiology and

Preventive Medicine,

Department of

Medicine, Monash

University/Alfred

Hospital, Prahran,

Victoria, Australia

H Krum

D Ninio

Heart/Lung

Transplantation

Service, St Vincent's

Hospital,

Darlinghurst, NSW,

Australia

P MacDonald

Correspondence to:

Dr Krum

henry.krum@

med.monash.edu.au

Accepted 16 August 2000 $\beta$ Adrenoceptor blockers are of well established clinical benefit when added to angiotensin converting enzyme (ACE) inhibitors and diuretics (with or without digoxin) in patients with New York Heart Association (NYHA) class II and III systolic chronic heart failure. ${ }^{12}$ However, despite the extensive clinical trial data supporting their use in this setting, the uptake of $\beta$ blockers has been relatively low. It is usually said that $\beta$ blockers are contraindicated for the treatment of systolic chronic heart failure because of the possibility that they might cause deterioration of cardiac function. Furthermore, certain $\beta$ blocking drugs such as carvedilol also possess vasodilator properties, ${ }^{3}$ which may add to physicians' concerns over the use of these agents in chronic heart failure.

These factors may make physicians reluctant to use agents such as carvedilol in this setting, despite the overwhelming evidence of clinical benefit. This reluctance might be alleviated if it was possible to predict in advance which patients with chronic heart failure are likely to have difficulty in tolerating $\beta$ blocker treatment. At present, the data available to address this issue are double blind, placebo controlled clinical studies of the efficacy of $\beta$ blockade..$^{4-8}$ However, no independent baseline predictors of poor tolerance to $\beta$ blockade have emerged from such studies.

The carvedilol in chronic heart failure clinical programme included an open label initiation of active treatment in both the American multicentre trial ${ }^{4}$ and the Australia/New Zealand trial, ${ }^{5}$ which may have led to a perception of bias in patient selection for the double blind phase of the trials. These considerations may have led some physicians to conclude that the clinical trial programme of $\beta$ blockers in chronic heart failure does not reflect the true value of these agents. There are very few reports on tolerance to $\beta$ blockade in everyday clinical practice outside of the controlled clinical trial environment. As carvedilol is the only 
$\beta$ blocker approved in Australia for use by specialist physicians in the treatment of systolic chronic heart failure (and has not been approved for any other indication), this gave us the opportunity to examine the above issues in a clinical practice setting.

Our aim in this study was therefore to determine which patients were able to tolerate the initiation and up-titration of carvedilol and be maintained on it in everyday clinical practice, as determined from baseline demographic and cardiac variables.

\section{Methods}

PATIENT POPULATION

This was a retrospective analysis of all patients with systolic chronic heart failure who were initiated on open label carvedilol treatment in hospital outpatient clinics (two sites) and cardiological private practice (five sites) throughout Australia since the drug was approved in 1997. A minimum of 30 consecutive patients initiated on carvedilol was required at each individual site. Patients receiving carvedilol for indications other than chronic heart failure were excluded from the analysis. Patients had to have been receiving carvedilol for at least three months in order to contribute to the study. The only exception was patients in whom carvedilol had been discontinued within three months of starting the treatment; these patients were included in the data analysis and reasons for discontinuation ascertained.

\section{STUDY DESIGN}

Baseline (pre-carvedilol) demographic and cardiac status data were collected. Specifically, data on the following baseline variables were collected: demographics (age, sex, height, weight); cardiac status (baseline blood pressure, heart rate, NYHA class, ejection fraction (within the previous 12 months); six minute walk test (within the previous three months); heart rhythm; and plasma sodium, urea, and creatinine concentrations. Also recorded was the duration of heart failure, the presence of a permanent pacemaker, a history of ventricular arrhythmia, the need for intravenous heart failure drug treatment in the previous three months, and hospital admission for heart failure in the previous three months.

Comprehensive data were also collected on patient comorbidities and potential causes of heart failure. Specifically, data were collected on the presence or absence of systemic hypertension, diabetes, ischaemic heart disease, myocardial infarction, and valvar heart disease, as well as on previous alcohol intake, drug treatment, and other factors that may have contributed to the development of chronic heart failure. In addition, data were collected on other diseases relevant to the initiation of $\beta$ blockade such as fixed airways disease, asthma, and peripheral vascular disease.

Data were collected on concomitant drug treatment taken by the patient at the time carvedilol was started. These included back- ground chronic heart failure drugs, with their dosage, other cardiovascular drugs, and noncardiovascular drugs.

Data were then obtained on the initiation and up-titration of carvedilol treatment. In particular, data were collected on the starting dose, the first stable carvedilol dose (defined as the first dose which was unchanged for three months), time to first stable carvedilol dose, and the ACE inhibitor and diuretic dose at the time of the first stable carvedilol dose.

Finally, data on current status of the patient were obtained-whether they were alive, dead, or had received a heart transplant. We were particularly interested in whether patients were on the drug (and the dose) at the time of death or transplantation, or whether they were alive and still on the drug. If patients were still taking carvedilol, the dose was recorded together with the dose of other concomitant drugs. If patients had stopped taking carvedilol, the dose at which the drug was discontinued and reasons for cessation were recorded.

\section{STATISTICAL ANALYSIS}

To address whether there were baseline differences between patients who tolerated carvedilol and those who did not, a proportional hazards regression model was fitted in which associations between baseline variables and time to discontinuation were tested. In the univariate analysis, each term was fitted separately, with time to discontinuation curves constructed and log rank tests performed. Continuous variables, for example blood pressure, were stratified by quartile in the proportional hazards regression model. Similarly, tolerance in relation to NYHA class was assessed by a proportional hazards analysis on time to discontinuation, with NYHA class fitted using indicator variables. Baseline variables that were univariate predictors of tolerance to carvedilol were subsequently fitted to a multiple proportional hazards regression model. A probability value of $p<0.05$ was considered to be significant.

\section{Results}

Eight hundred and eight patients were recruited into the study: $86.6 \%$ were male and $13.4 \%$ were female; $76 \%$ were recruited from hospital specialist heart failure clinics and $24 \%$ from private cardiological practice. Their mean age was 58 years (range 0-91 years). The patients mostly had NYHA class II and III symptoms of chronic heart failure (NYHA class I/II/III/IV, 59/201/254/89). Fifty patients $(6 \%)$ had died and $44(5 \%)$ had received a heart transplant. Six hundred and six patients $(75 \%)$ were current users of carvedilol and 95 $(12 \%)$ had stopped use but were still alive and had not had a heart transplant.

Thus carvedilol was "tolerated" as defined in this study (currently on carvedilol for a minimum three months of treatment, or on carvedilol at time of death or transplant) by $88 \%$ of patients. The mean first stable dose was $19 \mathrm{mg}$ twice daily (interquartile range 12.5$25 \mathrm{mg}$ twice daily). The mean time to first 
stable dose was 89 days (interquartile range 32-191 days).

BASELINE PREDICTORS OF TOLERANCE

Discontinuation of carvedilol was significantly related to NYHA class, with $97 \%$ of class I patients able to tolerate carvedilol but only $78 \%$ of class IV patients tolerating the drug (fig 1 ). Other baseline variables that were significant predictors of inability to tolerate carvedilol on univariate analysis were increased age, low diastolic blood pressure, and high blood urea concentration. Hazard ratios for inability to

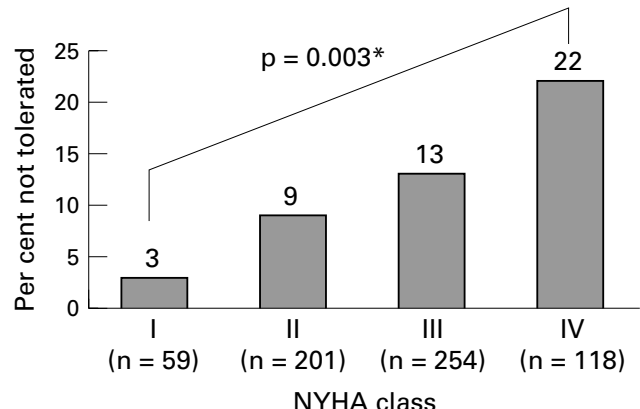

Figure 1 Per cent of patients unable to tolerate carvedilol treatment, grouped according to New York Heart Association (NYHA) functional class. Data analysed by proportional hazards analysis on time to discontinuation (intolerance), with NYHA class fitted using indicator variables. * Proportional hazards analysis on time to discontinuation (intolerance) with NYHA class fitted using indicator variables.

Table 1 Selected demographic variables in patients who were able to tolerate or could not tolerate carvedilol

\begin{tabular}{lcll}
\hline & Tolerated & Not tolerated & Mean \\
\hline Male/female (\%) & $86.6 / 13.4$ & $86.6 / 13.4$ & $86.6 / 13.4$ \\
Age (years) & 57.6 & 58.5 & 57.8 \\
LVEF (\%) & 27.1 & 28.2 & 27.2 \\
DBP (mm Hg) & 73.9 & $70.1^{\star}$ & 73.4 \\
Heart rate (beats/min) & 81.8 & 79.2 & 81.5 \\
Serum Na ${ }^{+}(\mathrm{mmol} / \mathrm{l})$ & 138.6 & 138.2 & 138.6 \\
Plasma urea (mmol/1) & 9.6 & 15.4 & 11.3 \\
ACE inhibitor (\%) & 87.7 & 80.2 & 86.6 \\
\hline
\end{tabular}

Only low diastolic blood pressure was a significant univariate predictor of poor tolerability.

${ }^{\star} \mathrm{p}<0.05$ between group.

$\mathrm{ACE}$, angiotensin converting enzyme; DBP, diastolic blood pressure; LVEF, left ventricular ejection fraction.

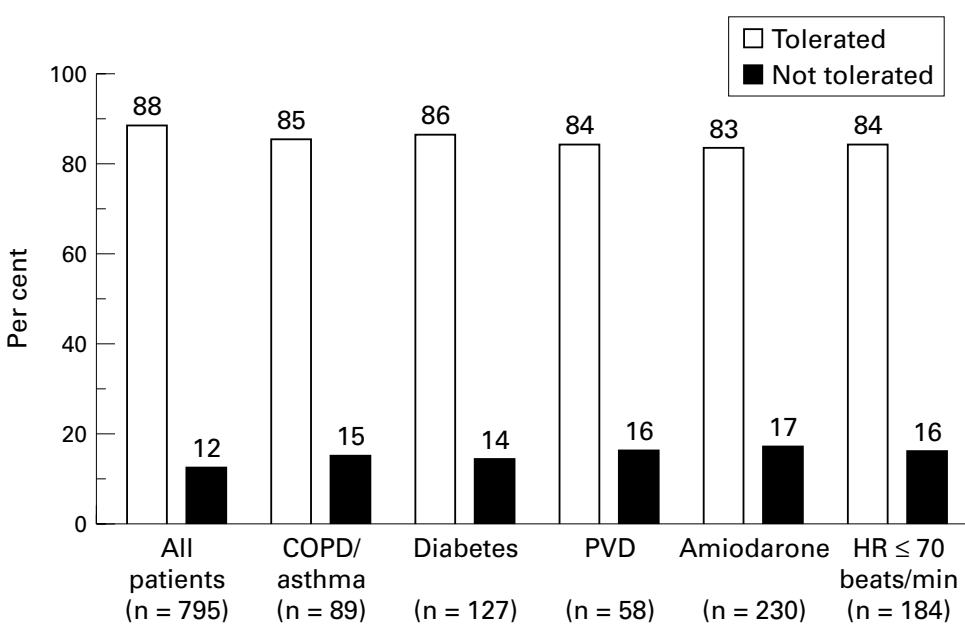

Figure 2 Per cent of patients able to tolerate carvedilol treatment, grouped according to traditional contraindications and precautions in prescribing a $\beta$ blocker. $C O P D$, chronic obstructive pulmonary disease; HR, heart rate; PVD, peripheral vascular disease. tolerate carvedilol were: increased age in years, 1.01 (95\% confidence interval (CI) 1.0 to 1.3 ); low diastolic blood pressure, 1.04 (95\% CI 1.02 to 1.08 ); and high urea concentration, 1.04 (95\% CI 1.02 to 1.05 ). However, when these univariate predictors of poor tolerance were fitted into a multivariate regression equation, no single independent predictor of poor tolerance emerged.

TOLERANCE ACCORDING TO DEMOGRAPHIC DATA Demographic data in patients who were able to tolerate or could not tolerate carvedilol are summarised in table 1. Only diastolic blood pressure was a significant predictor of poor tolerance.

TOLERANCE ACCORDING TO PRESCRIBING PHYSICIAN

We categorised tolerance according to hospital heart failure specialist or private cardiology practitioner as managing physician. Among hospital specialists (603 patients), $87 \%$ of the patients tolerated carvedilol and $13 \%$ did not. Among private practitioners (192 patients), $92 \%$ of the patients tolerated carvedilol and $8 \%$ did not. We also compared tolerance among the first $50 \%$ of patients treated by practitioners - that is, the earliest experience of carvedilol-compared with the second 50\%. More patients tolerated carvedilol among the second $50 \%(91 \% v 86 \%)$ but the difference was not significant by $\chi^{2}$ analysis.

TOLERANCE ACCORDING TO TRADITIONAL

PRECAUTIONS IN PRESCRIBING $\beta$ BLOCKERS

We were able to examine tolerance to carvedilol in relation to traditional relative contraindications to prescribing $\beta$ blockers. These included chronic airways disease/asthma, diabetes, peripheral vascular disease, low heart rate, and the use of concomitant agents that may exacerbate adverse effects with $\beta$ blockade, for example amiodarone. These data are summarised in fig 2 .

OTHER DATA ON CARVEDILOL TOLERANCE A breakdown of the first stable dose of carvedilol is presented in fig 3. Most patients were able to reach the standard target dose of $25 \mathrm{mg}$ twice daily.

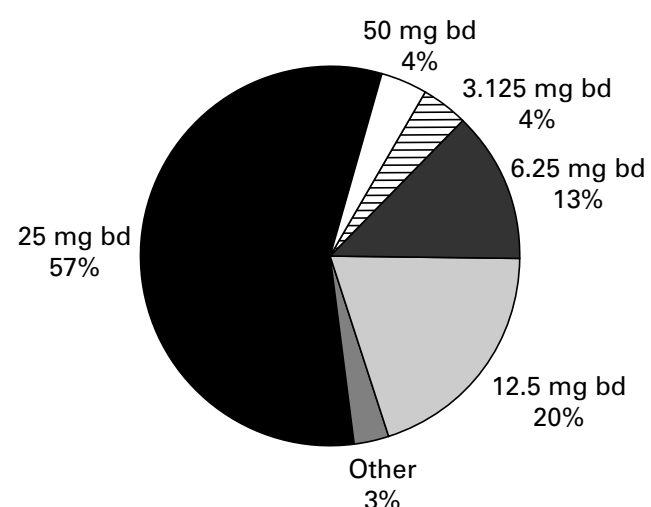

Figure 3 First stable dose of carvedilol achieved in study patients. 


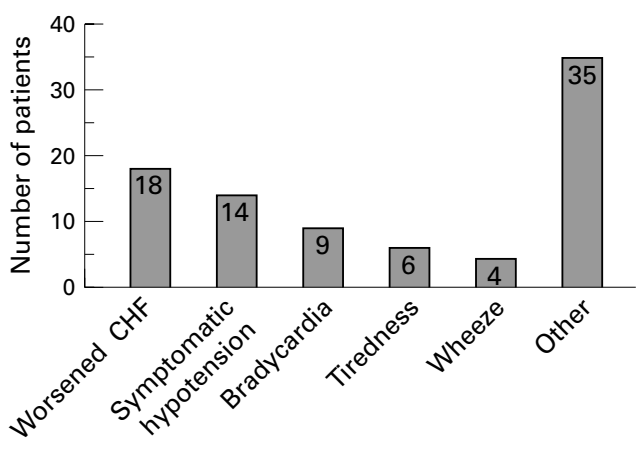

Figure 4 Reason for discontinuation of carvedilol among study patients. CHF, chronic heart failure.

Reasons for discontinuation of carvedilol are shown in fig 4 . These include expected reasons for discontinuation such as worsened heart failure, symptomatic hypotension and bradycardia, as well as tiredness and wheeze. A series of other reasons for discontinuation comprise a large proportion of the subset. These included cough, nausea, arrhythmia, renal dysfunction, diarrhoea, rash, and non-compliance.

\section{Discussion}

In this study we sought to examine baseline variables that may predict tolerance to carvedilol in patients with chronic heart failure when the drug was initiated during everyday clinical management. We specifically sought to do this outside the clinical trial setting, as this drug is now approved for heart failure management and is widely prescribed. Furthermore, carvedilol is the only $\beta$ blocker currently approved for use in systolic chronic heart failure in Australia, and it is not approved for any other indication. Thus it may be expected that an assessment of tolerance among all patients with systolic chronic heart failure who were prescribed this drug in everyday practice would closely reflect physicians' experience with initiating $\beta$ blockade in this setting. The opportunity to examine tolerance during ordinary use (in contrast to during the conduct of clinical efficacy trials) would be expected to be particularly relevant for the prescribing physician.

Overall, our findings were that initiation of $\beta$ blockade with carvedilol was extremely well tolerated. The tolerance observed among patients prescribed the drug in the private practice setting was at least as good as, if not better than, that seen in the hospital setting. It must be emphasised, however, that this study excluded analysis of patients who may have been eligible to receive carvedilol treatment but who were not started on the drug by their treating physician. As we did not analyse such patients, it was not possible to assess the degree of patient selection undertaken by the physicians contributing patients to this study. However, in the recent BRING-UP study ( $\beta$ blockers in patients with congestive heart failure: guided use in clinical practice), ${ }^{9}$ a "real world" study conducted in Italy, $47.2 \%$ of patients considered for $\beta$ blockade did not ultimately receive these agents.

Very few baseline variables predicted the inability of patients to tolerate carvedilol.
Those that were predictive statistically on univariate analysis (advanced age, low diastolic blood pressure, high urea, and advanced NYHA class) are to be expected, on the basis that they reflect patients more vulnerable to the effects of cardiovascular drugs in general (advanced age), those with more severe disease (low diastolic blood pressure and advanced NYHA class), or those with multiple organ impairment (high urea concentration). Because these factors tend to cluster within certain individuals (colinearity), it was not surprising that none of the baseline variables that were predictive on univariate analysis remained as independent predictors when entered into a multivariate model.

The finding that no baseline variable could predict inability to tolerate $\beta$ blocker treatment is consistent with data from recent clinical trials which were also generally unable to find baseline predictors of poor tolerance. ${ }^{4-8}$

An important finding in the present study was that patients with comorbidities that would represent a relative contraindication to $\beta$ blocker treatment were in fact able to tolerate carvedilol remarkably well. These comorbidities include diabetes, chronic airways obstruction, and peripheral vascular disease, as well as other relative contraindications to $\beta$ blocker treatment. There was very little further reduction in the percentage of patients able to tolerate carvedilol in comparison with the cohort as a whole. These findings may reflect a degree of patient selection in that physicians will only have prescribed the drug to patients whom they thought capable of tolerating $\beta$ blocker treatment. This is supported by the observation in the BRING-UP study ${ }^{9}$ that the main reason for non-selection of a patient for $\beta$ blockade is a perceived contraindication to giving the drug. Nevertheless the findings of our present study suggest that if a clinically guided selection of patients with such comorbidities is made by the treating physician, tolerance to the drug can be expected to be reasonably good.

Because $\beta$ blocker treatment is up-titrated from relatively low doses in the management of patients with chronic heart failure, another marker for tolerance to these agents is the first stable dose achieved. In this analysis, $61 \%$ of patients were able to reach a dose of carvedilol of $25 \mathrm{mg}$ twice daily or more, again reflecting excellent tolerance of the drug. Thus only a minority of patients were unable to reach the target dose of treatment. Indeed, all but $4 \%$ of patients were able to reach $6.25 \mathrm{mg}$ twice daily, which is where clinical benefit begins to be observed with the drug. ${ }^{10}$ The figures on the percentage reaching target dose compare favourably with those observed with ACE inhibitors in clinical trials ${ }^{11}$ and in everyday practice. ${ }^{12}$

\section{CONCLUSIONS}

Carvedilol treatment for systolic chronic heart failure was extremely well tolerated in this retrospective analysis of carvedilol initiation, up-titration, and ongoing treatment. The majority of patients were able to achieve target doses of drug, and the tolerance observed in the hospital setting was preserved in private 
practice. Furthermore, there was little fall off in tolerance among selected patients with relative contraindications to $\beta$ blocker treatment. Univariate predictors of poor tolerance reflected a general increase in the risks of receiving cardiovascular drugs or having more advanced disease. However, on multivariate analysis there were no independent predictors of inability to tolerate carvedilol treatment.

Supported by a grant from F Hoffmann-LaRoche Ltd, Basel, Switzerland. The study was conceived, designed, and conducted by the authors, independent of the study sponsors. We wish to acknowledge the expert statistical support of $\mathrm{Dr}$ Andrew Martin, Roche Biometrics, Dee Why, NSW, Australia, in assisting with analysis of this study. Study sites: Victoria (H Krum, D Ninio, D Rose), NSW (P MacDonald), Tasmania (M Loughead), Western Australia (M Ireland). Study Coordinators: J Hankin, T James, J Ferguson.

1 Task Force of the Working Group on Heart Failure of the European Society of Cardiology. The treatment of heart failure. Eur Heart f 1997;18:736-53.

2 Advisory Council to Improve Outcomes Nationwide in Heart Failure. Consensus recommendations for the management of chronic heart failure. $\mathrm{Am} f$ Cardiol 1999;83:2-38A

3 Ruffolo RR, Gellai M, Hieble JP, et al. The pharmacology of carvedilol. Eur F Clin Pharmacol 1990;38:S82-8.
4 Packer M, Bristow MR, Cohn JN, et al, for the US Carvedilol Heart Failure Study Group. The effect of carvedilol on morbidity and mortality in patients with chronic heart failure. N Engl f Med 1996;334:1349-55.

5 Australia-New Zealand Heart Failure Research Collaborative Group. Randomized, placebo-controlled trial of carvedilol in patients with congestive heart failure due to ischemic heart disease. Lancet 1997;349:375-80.

6 MERIT-HF Study Group. Effect of metoprolol CR/XL in chronic heart failure: metoprolol CR/XL randomised intervention trial in congestive heart failure. Lancet 1999;353:2001-7.

7 CIBIS Investigators. A randomized trial of $\beta$-blockade in heart failure: the Cardiac insufficiency bisoprolol study. Circulation 1994;90:1765-73.

8 CIBIS II Investigators and Committees. The cardiac insufficiency bisoprolol study (CIBIS-II): a randomised trial. Lancet 1999;353:9-13.

9 Balli E, Lucci D, Gorini M, et al. Clinical variables predicting the use of beta-blockers in heart failure: the BRING-UP study [abstract]. Eur Heart $\mathcal{F}$ 1999;20(suppl):P624.

10 Bristow M, Gilbert EM, Abraham WT, et al, for the MOCHA investigators. Carvedilol produces dose-related improvements in left ventricular function and survival in subjects with chronic heart failure. Circulation 1996;94:2807-16.

11 The SOLVD Investigators. Effect of enalapril on survival in patients with reduced left ventricular ejection fractions and congestive heart failure. N Engl f Med 1991;325:293-302.

12 Krum H, Tonkin A, Currie R, et al. Pharmacological management of chronic heart failure in Australian general practice: the cardiac awareness survey and evaluation (CASE) study. Proceedings of the $47^{\text {th }}$ Annual Scientific Meeting of the Cardiac Society of Australia and New Zealand, Wellington, 1999: abstract 306

\section{IMAGES IN CARDIOLOGY}

\section{Silent patent ductus arteriosus endarteritis}

An 18 year old woman, without a previous history of cardiovascular disease, was referred for ultrasound examination because of pyrexia during the previous 40 days. Laboratory investigations produced three blood cultures with Streptococcus sanguis, while cardiac auscultation revealed only a soft systolic murmur at the second left intercostal space.

Echocardiographic examination showed normal cardiac dimensions and function, normal

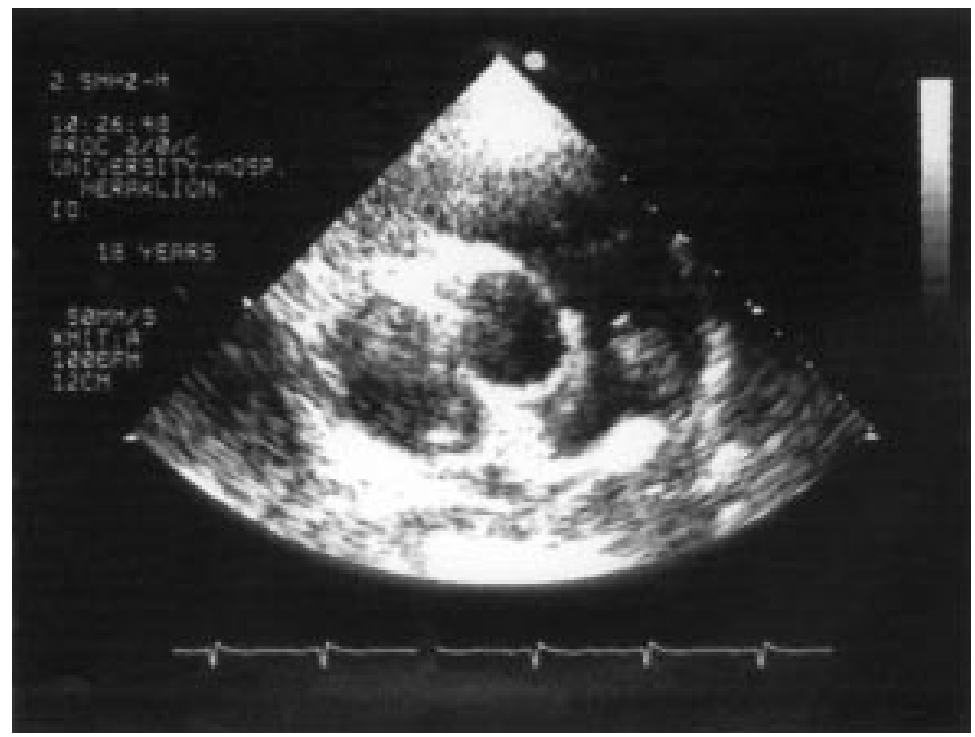

valves with no vegetations, and no signs of pulmonary hypertension. In a modified short axis view at the level of the great arteries colour Doppler flow mapping revealed a tiny jet of retrograde flow in the main pulmonary area, indicative of patent ductus arteriosus (PDA).

A large mobile mass was noted at the confluence of the left pulmonary artery, consistent with a vegetation at the entrance of the PDA (left). The discovery of this mass in a patient with streptococcus septicaemia led us to the diagnosis of PDA endarteritis.

Following successful antibiotic treatment, surgery confirmed the presence of PDA; the ductus was closed and the mass was removed. Histological examination confirmed that the latter was vegetation.

Estimation of the risk of infective endarteritis in patients with PDA is an important issue that affects clinical decision making. The classic continuous murmur is usually sufficient in itself for repair, in part to eliminate the risk of infective endarteritis. Although endarteritis may appear as a complication of clinically apparent PDA, it is not generally found in silent PDA, and its treatment with routine antibiotic prophylaxis and surgical closure is under discussion. However, as shown by our case, only the second such to be reported, the risk of infection still exists even in silent PDA. 